\title{
1-D polymer ternary composites: Understanding materials interaction, percolation behaviors and mechanism toward ultra-high stretchable and super- sensitive strain sensors
}

\author{
Senlong Yu, Xingping Wang, Hengxue Xiang*, Mike Tebyetekerwa and Meifang Zhu*
}

\begin{abstract}
A series of 1-D polymer ternary composites based on poly(styrene-butadiene-styrene) (SBS)/carbon nanotubes (CNTs)/few-layer graphene (FLG) conductive fibers (SCGFs) were prepared via wet-spinning. Employed as ultra-high stretchable and super-sensitive strain sensors, the ternary composite fiber materials' interaction, percolation behaviors and mechanism were systematically explored. The resultant SCGFs-based strain sensors simultaneously exhibited high sensitivity, superior stretchability (with a gauge factor of 5,467 under $600 \%$ deformation) and excellent durability under different test conditions due to excellent flexibility of SBS, the synergistic effect of hybrid conductive nanofibers and the strong $\pi-\pi$ interaction. Besides, the conductive networks in SBS matrix were greatly affected by the mass ratio of CNTs and FLG, and thus the piezoresistive performances of the strain sensors could be controlled by changing the content of hybrid conductive fillers. Especially, the SCGFs with $0.30 \mathrm{wt} . \%$ CNTs (equal to their percolation threshold $0.30 \mathrm{wt} . \%$ ) and $2.7 \mathrm{wt} . \%$ FLG demonstrated the highest sensitivity owing to the bridge effect of FLG between adjacent CNTs. Whereas, the SCGFs with $1.0 \mathrm{wt} . \%$ CNTs (higher than their percolation threshold) and $2.0 \mathrm{wt} . \%$ FLG showed the maximum strain detection range $(600 \%)$ due to the welding connection caused by FLG between the contiguous CNTs. To evaluate the fabricated sensors, the tensile and the cyclic mechanical recovery properties of SCGFs were tested and analyzed. Additionally, a theoretical piezoresistive mechanism of the ternary composite fiber was investigated by the evolution of conductive networks according to tunneling theory.
\end{abstract}

Keywords: strain sensor, poly(styrene-butadiene-styrene), carbon nanotube, few-layer graphene, wet-spinning

\section{INTRODUCTION}

Rubber-like conductive polymer nanocomposites can be applied in the field of flexible strain sensors such as human body sign detection, human-computer interaction and virtual reality where the resistivity signal changes with the deformation of conductive polymers [1-3]. According to the previous reports [4], some characteristics such as high sensitivity, flexibility, stability and light weight are essential for stretchable strain sensors. However, the strain sensors reported so far are not able to meet all the listed requirements simultaneously. For example, the sensors based on polyurethane acrylate and cracked Pt exhibited a high gauge factor (GF) of about 2,000 at $2 \%$ strain [5]. Moreover, the sensitivity of this nanoscale crack-based sensor could be further enhanced by controlling the crack depth, exhibiting a superior GF value of 16,000 at $2 \%$ strain [6]. However, the sensors could not meet the requirements for the deformation highly required in the human body sign detection even if their sensitivity was high. Conversely, the stretchable strain sensors have been also reported using ultrathin gold nanowires and carbon nanomaterials, which exhibit wide work range $(>100 \%)$ but limited sensitivity $(\mathrm{GF}<10)$ $[7,8]$. In addition to high sensitivity and stretchability, the dynamic durability is also an important characteristic index for flexible sensors $[9,10]$. In most cases, the deterioration of durability is relevant to the irreversible deformation of the flexible matrix, the fracture of conductive network and weak interaction between polymer and conductive fillers [11-13]. Aiming at the problems above, we recently prepared strain sensors based on poly(styrene-butadiene-styrene) (SBS)/carbon

State Key Laboratory for Modification of Chemical Fibers and Polymer Materials, College of Materials Science \& Engineering, Donghua University, Shanghai 201620, China

*Corresponding authors (emails: hengxuexiang@dhu.edu.cn (Xiang H); zhumf@dhu.edu.cn (Zhu M)) 
nanotubes (CNTs) and SBS/few-layer graphene (FLG) conductive fibers, which possessed large deformation, high sensitivity and good cycle stability under small deformation $(<50 \%)[14,15]$. Unfortunately, the dynamic stabilities of these hybrid conductive fibers is low under large deformation $(>100 \%)$, which limited their application in the fields of human motion detection and smart wearable devices.

Recently, it was reported that the conductive network and strain sensing performance can be efficiently improved by hybrid conductive fillers $[16,17]$. Lin et al. [18] reported that nickel powders with high tunnel barriers could increase the tensile strain sensitivity of polyurethane (PU)/CNTs composites, and carbon black (CB) could inhibit CNTs entanglement and accelerate the destruction of conductive network under the tensile where a higher tensile strain sensitivity was presented in composite materials [19]. Lee et al. [20] discovered that oriented silver nanowires could act as bridges between silver nanoparticles under tension state, exhibiting high conductivity retention of composite fibers under high strain. In addition, the deterioration of mechanical properties for strain sensors could be dramatically avoided due to low percolation threshold caused by the synergistic effect of hybrid conductive fillers. Ke et al. [21] manufactured a sensor based on $\mathrm{PVDF} / \mathrm{CB} / \mathrm{CNTs}$ composite, in which the higher the $\mathrm{CB}$ amount used, the higher the response sensitivity of sensors and the lower electroosmotic flow threshold. In this scenario, Liu et al. [22] fabricated flexible strain sensors based on $\mathrm{PU} / \mathrm{CNT}$ /FLG conductive composites. As expected, the entanglement between CNTs would be inhibited by the graphene spacer, and thus the dispersion of CNTs and conductive network in polymer matrix were improved. At the same time, CNTs could be used as bridges to connect adjacent graphene sheets. Unfortunately, such composite materials present poor stability under high applied strain.

In this work, SBS/CNTs/FLG hybrid conductive nanofibers were prepared via a facile and scalable wetspinning process to fabricate high performance strain sensors. The effects of hybrid conductive fibers on morphology, mechanical property, hysteresis effect and piezoresistive performance of the strain sensors were investigated using stretching-releasing cycle tests under different mass ratios of CNTs/FLG, applied strains and strain rates. Besides, the sensing mechanism of the ternary composite fibers was also well explained by the evolution of conductive network according to tunneling theory.

\section{EXPERIMENTAL SECTION}

\section{Materials}

SBS triblock copolymer $\left(\rho=0.94 \mathrm{~g} \mathrm{~cm}^{-3}\right)$ with $28 \mathrm{wt} . \%$ styrene was purchased from Kraton Corporation (Texas, USA). Multiwalled CNTs (NC7000) with an average diameter about $9.5 \mathrm{~nm}$ and a length of $1.5 \mu \mathrm{m}$ was obtained from Nanocyl S.A. (Belgium). FLG (G-100) with $7.5 \mathrm{wt} . \%$ total oxygen content and about 700-1,500 S m${ }^{-1}$ conductivity was supplied by Shanghai Simbatt Energy Technology Co. Ltd (China). The morphologies and structures of CNTs and FLG were shown in Fig. S1 (Supplementary information). All other chemical reagents were purchased from Sinopharm Chemical Reagent Co., Ltd. (China).

\section{Preparation of SBS/CNTs/FLG composite fibers}

The SBS/CNTs/FLG composite fibers (SCGFs) were fabricated by a facile and scalable wet-spinning process $[14,15]$. First, a certain proportion of CNTs and FLGs were added into tetrahydrofuran solution for a further ultrasonic of $4 \mathrm{~h}$ in a $20^{\circ} \mathrm{C}$ water bath to obtain a dispersed mixed solution. Then, a given mass of SBS was fully dissolved in the mixed solution where the total mass fraction of SBS, CNTs and FLG in the solution was controlled to be $15 \mathrm{wt} . \%$. And then, SBS/CNTs/FLG composite fibers could be fabricated after extruding the mixed solution into ethanol coagulation using a homemade spinning equipment where the spinning liquid flow rate was $6 \mathrm{~mL} \mathrm{~h}^{-1}$ and the spinneret's diameter was $330 \mu \mathrm{m}$. The resultant SCGFs are denoted as SBS $/ x \mathrm{C} / y \mathrm{G}$, where $x$ and $y$ represent the mass percentage of CNTs and FLG in the composite fibers, respectively. Taking SBS/1C/ $2 \mathrm{G}$ as an example, it represents that CNTs content and FLG content in the composite fibers are $1.0 \mathrm{wt} . \%$ and $2.0 \mathrm{wt} . \%$, respectively.

\section{Characterization}

Dispersion of CNTs and FLG in SBS: a field emission electron microscope (JEOL JSM-4800LV, Hitachi, Japan) was adapted to observe the morphology and microstructure of the composite fibers. The fiber cross-sections could be obtained after SCGFs were quenched in liquid nitrogen for $2 \mathrm{~min}$. All samples were spray-coated with platinum before observation.

Mechanical performance evaluation of SCGFs: the tensile mechanical properties of the fibers were tested with an Instron electronic universal test system (Model 5996, USA). The fiber grip length was $10 \mathrm{~mm}$ and the stretching rate was $200 \% \mathrm{~min}^{-1}$. Five groups were tested 

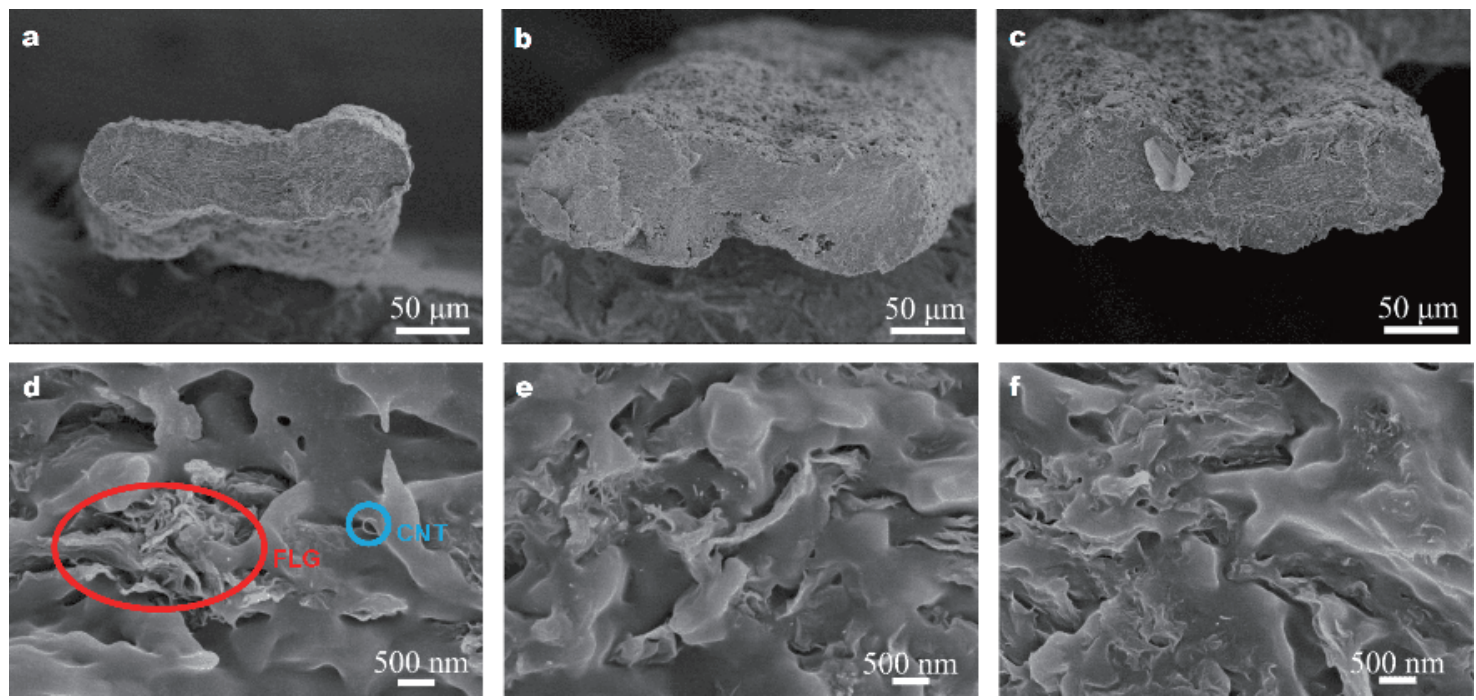

Figure 1 Cross-sectional SEM images of SCGFs: (a) SBS/0.3C/2.7G, (b) SBS/0.5C/2.5G, (c) SBS/1C/2G, (d-f) are the higher magnification of (a-c), respectively.

for each sample, and the analytical data was average.

Piezoresistive performance of SCGFs: the tensile strain tester was constituted with a tensile strain measurement system (electronic universal testing machine, Instron 5996), a resistance test system (digital multimeter, Keithley 2000) and a data collection system. The changes in fiber resistance were measured under different tensile strain rates, tensile strain amplitudes and tensile cycles. The specific operation was described according to our previous work $[14,15]$.

\section{RESULTS AND DISCUSSION}

\section{Morphology and structure of SCGFs}

The morphologies and structures of SCGFs were investigated by cross-sectional SEM images.In Fig. 1a-c, the cross-section of SCGFs is flat, owing to a wet spinning process where the diffusion rate of coagulating agent ethanol into fiber composite was higher than that of solvent (tetrahydrofuran) into coagulation bath [23]. Fig. $1 \mathrm{~d}-\mathrm{f}$ are the high-resolution and cross-sectional SEM images of SBS/0.3C/2.7G, SBS/0.5C/2.5G and SBS/ $1 \mathrm{C} / 2 \mathrm{G}$ composite fibers. As shown in the images, FLG loosely accumulates together and uniformly disperses in the SBS matrix (red oval), while CNTs also distribute on the cross-sections of these composite fibers (blue oval). The FLG and CNTs are completely embedded in SBS, indicating a strong interaction and good interfacial compatibility between FLG, CNTs and SBS. The main reason is th e strong $\pi-\pi^{*}$ interaction between benzene rings in FLG, CNTs and SBS [24,25], while FLG surface with folds could increase the mechanical interlocking with SBS molecule chains and further enhance the adhesion between multiple components [26].

\section{Mechanical behaviors of SCGFs}

The fibers with high tensile mechanical strength and excellent elastic recovery are essential for strain sensors. As shown in Fig. 2, the unidirectional tensile strength of pure SBS, SBS/0.3C/2.7G, SBS/0.5C/2.5G and SBS/1C/2G composite fibers are $32.8,14.3,21.3$ and $17.0 \mathrm{MPa}$, with the elongation at break of $1,330 \%, 870 \%, 949 \%$ and $700 \%$, respectively. Therefore, all of the SCGFs present good tensile mechanical properties and the elongation at break are all more than $700 \%$, which meets the tensile performance requirements of strain sensors under large deformation. Moreover, the mechanical property of SBS/ $0.5 \mathrm{C} / 2.5 \mathrm{G}$ is better than SBS/3CNT and SBS/3FLG $[14,15]$, which could be attributed to a synergistic effect between one-dimensional CNTs and two-dimensional FLG at this ratio, promoting uniform dispersion in the polymer matrix.

The elastic recovery and the mechanical hysteresis of the conductive composite fibers directly determine the response reproducibility and detection reliability of strain sensors. Therefore, the hysteresis effect of SCGFs was investigated using cyclic loading-unloading tests under the applied strain ranging from $50 \%$ to $600 \%$ at the strain rate of $200 \% \mathrm{~min}^{-1}$. The elastic recovery rate under different tensile strain amplitude could be calculated 

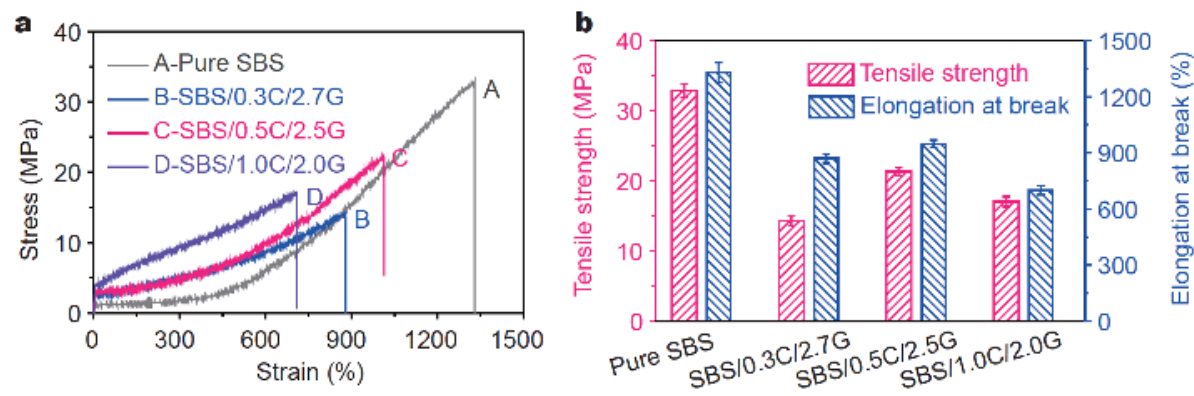

Figure 2 (a) Stress-strain curves of SCGFs with different mass ratios of CNTs to FLG. (b) Mechanical properties of the SCGFs.

based on Equation (1) [27], where $\varepsilon$ is the tensile strain, and $\varepsilon_{\mathrm{R}}$ is the residual strain after tensile-recovery tests.

$\mathrm{ER}_{\varepsilon}=\frac{\varepsilon-\varepsilon_{\mathrm{R}}}{\varepsilon} \times 100$.

In Fig. 3a, b, the elastic recovery rate of SCGFs increases with the increase of tensile strain and $m_{\mathrm{CNTs}} /$ $m_{\mathrm{FLG}}$ under the same tensile strain amplitude. As shown in Fig. 3c, d, the stress-strain curves of tensile phase and recovery phase in each stretching-releasing cycle are not coincident under the same tensile strain, indicating a hysteresis effect in SBS/1C/2G composite fibers. The mechanical hysteresis and the stress softening of SCGFs, which is the most obvious in the first cycle, reduce with the increase of cycle index. The mechanical hysteresis and the stress softening increase with the increase of tensile strains which is caused by the slippage between FLGs, FLG and SBS molecular chains, and SBS molecular chains under large applied strains [28].

\section{Electromechanical properties of SCGFs}

Effect of CNTs and FLG loading on the strain sensing behavior

The dosage and proportion of hybrid conductive fillers are important to the tensile strain response of strain sensors $[19,21]$. The relation curves of CNTs/FLG dosage changes on the responsive sensitivity and tensile strain of SCGFs under $50 \% \mathrm{~min}^{-1}$ tensile rate are shown in Fig. $4 \mathrm{a}, \mathrm{b}$. The sensitivity of strain sensors can be quantified by $\Delta R / R_{0}$ and GF. GF is defined as the ratio of
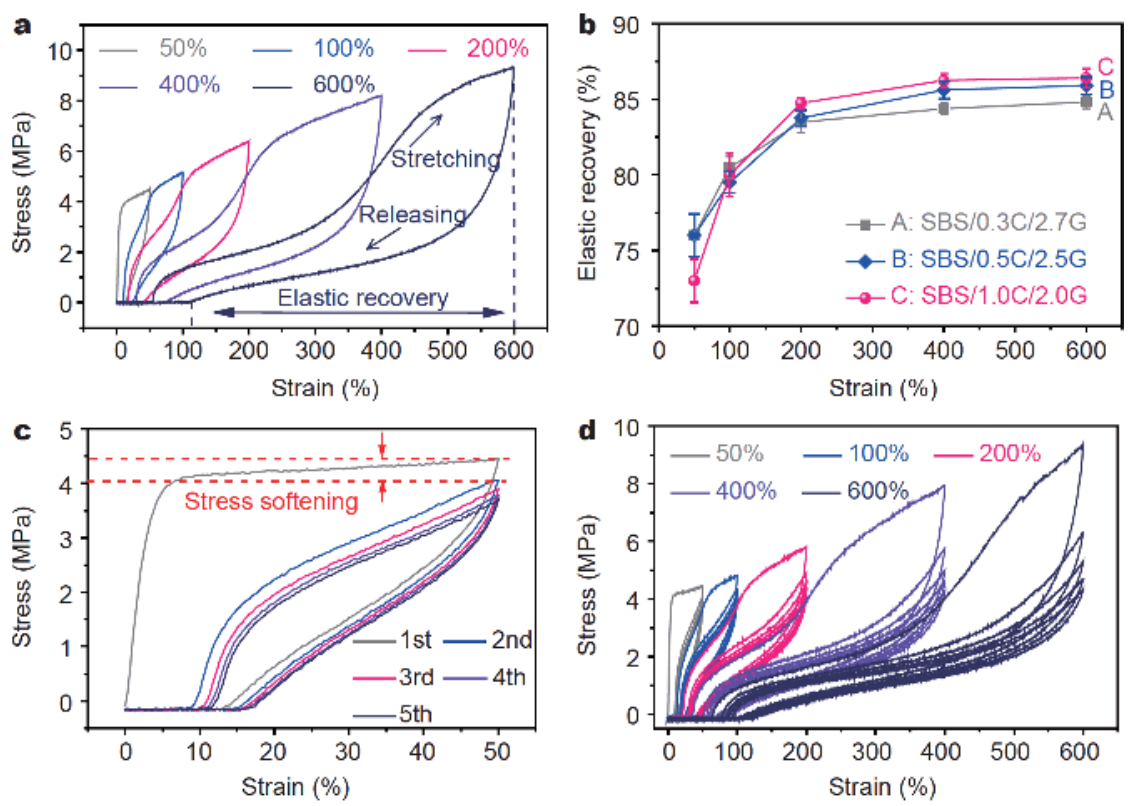

Figure 3 (a) Stress-strain curves of SBS/1C/2G composite fibers in cyclic elastic tests with growing maximal strain from 50\% 600\%. (b) Elastic recovery of SCGFs during cyclic stretching-releasing tests as a function of growing maximum strain. (c) Mechanical hysteresis of $50 \%$ strain for 5 cycles of SBS/1C/2G fiber. (d) Mechanical hysteresis for $50 \%, 100 \%, 200 \%, 400 \%$ and $600 \%$ strain of SBS/1C/2G fibers. 

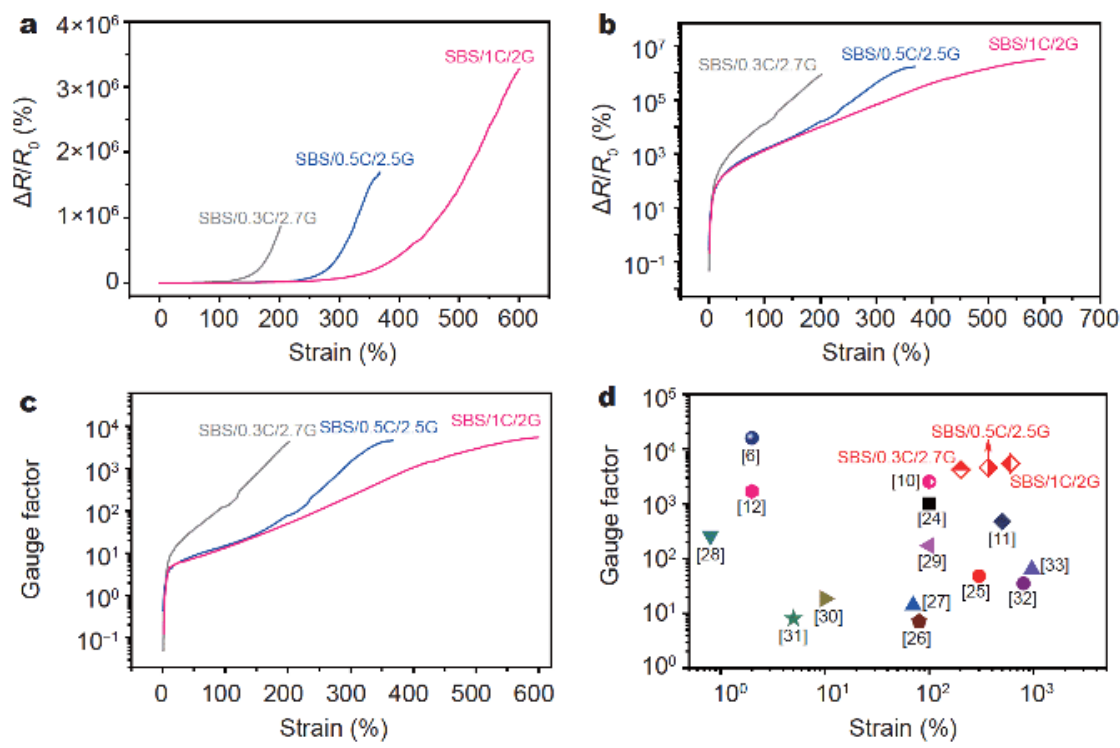

Figure 4 (a, b) $\Delta R / R_{0}$ of SCGFs as a function of applied strain. (c) GF of SCGFs as a function of applied strain. (d) Comparison of the performance of the SCGFs sensor with that of recently reported flexible strain sensors. Note: $\Delta R / R_{0}$ and GFs of (b-d) are in logarithmic coordinates.

$\Delta R / R_{0}$ to the applied strain $\left(\Delta R / R_{0}\right) / \varepsilon$, where $\Delta R$ is the change in resistance, $R_{0}$ is the initial resistance, and $\varepsilon$ is the applied strain. As shown in Fig. 4b, c, the SCGFs exhibit a "positive strain effect" during the stretching process. The response sensitivity of the composite fibers increases with the increase of tensile strains, and the GF of composite fibers decreases with the increase of $m_{\mathrm{CNTs}} s$ $m_{\mathrm{FLG}}$ under the same strains. Based on our previous studies [14], the percolation thresholds of SBS/CNTs and SBS/FLG composite fibers are $0.30 \mathrm{wt} . \%$ [15] and 2.7 wt.\%, respectively. Therefore, the CNTs and FLG contents in SBS/0.3C/2.7G composite fibers are near the percolation zone where the conductive network is sensitive to the external stimuli, exhibiting a high tensile strain response sensitivity coefficient. For SBS/1C/2G composite fibers, CNTs content was far higher than their percolation threshold, and thus a dense conductive network forms as a dense CNT network in this case and a synergistic effect of FLG, presenting better stability but a lower sensitivity coefficient under tensile strains. In Fig. 4d, the GFs of SCGFs in the maximum strain detection range exhibit certain advantages, showing a wide strain range and a high sensitivity coefficient [11,16,29-37]. Besides, the workable range and GFs (under the maximum applied strain) of SCGFs are superior to those of SBS/CNTs or SBS/FLG composite fibers (Table S1), which is beneficial to detecting large deformation. Furthermore, the response time of the SCGF-based sensor was further determined to be less

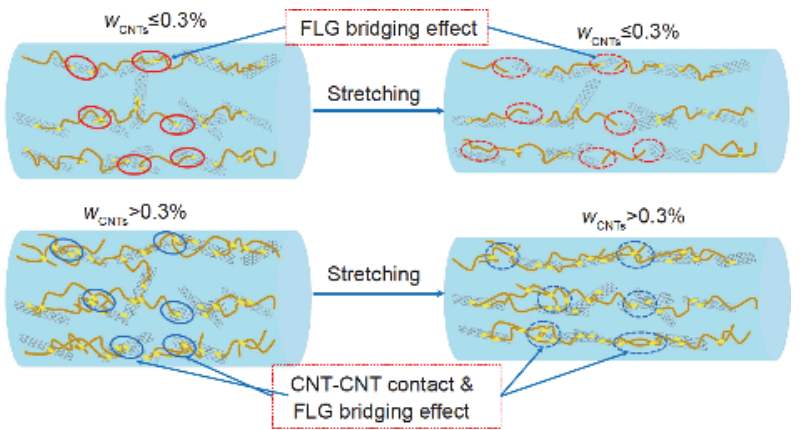

Figure 5 Schematic illustration of the conductive network consisting of CNTs and FLG before and after stretching for SCGFs with CNTs contents below and above $0.30 \mathrm{wt} . \%$.

\section{than 190 ms (Fig. S2).}

Based on the above analysis, the structural evolution diagram of the composite conductive network of CNTs and FLG in SCGFs during the stretching process was shown in Fig. 5. When the CNTs content was equal to their percolation threshold $(0.30 \mathrm{wt} . \%)$, the CNTs were not sufficient to form a complete conductive path, and FLG could bridge to connect CNTs into a conductive path (red region). During the stretching process, CNTs and FLG lost their connection or the gap increased due to the deformation of the matrix, leading to a sharp increase in the tunnel resistance and SCGFs resistance. When CNTs content in fibers was higher than their percolation threshold, CNTs were sufficient to form a continuous conductive path, and FLG further strengthened the 

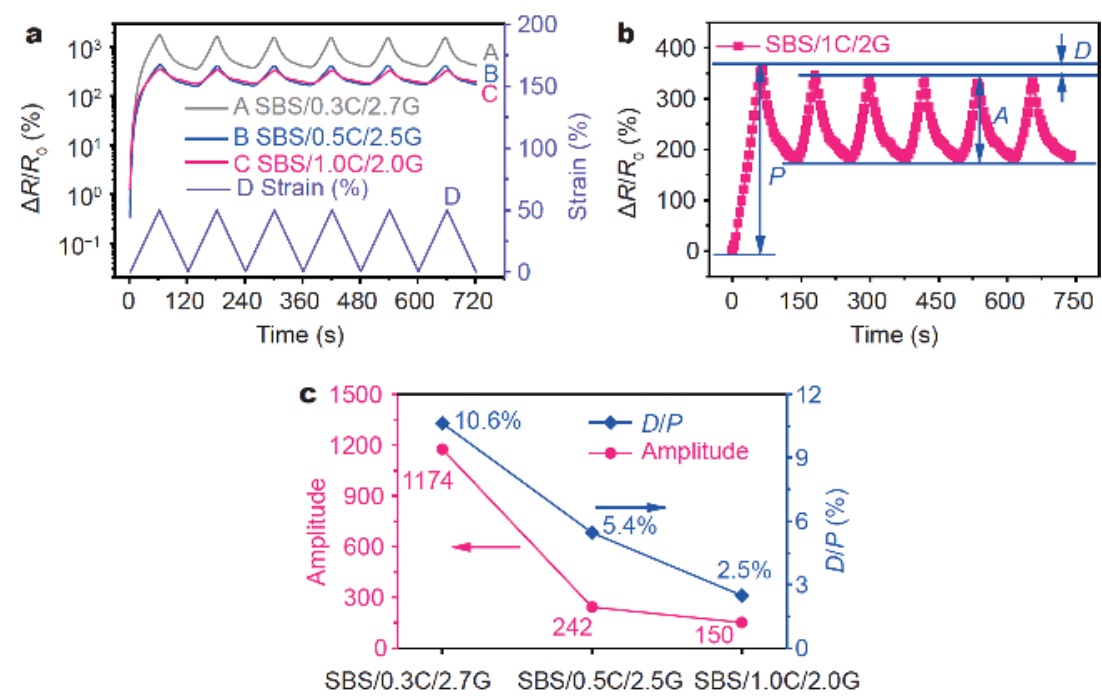

Figure 6 (a) The dynamic strain sensing behaviors of SCGFs under cyclic stretching and releasing at a strain of $0-50 \%\left(\right.$ Note: The $\Delta R / R_{0}$ is in logarithmic coordinates). (b) The representation of where recovery ratio $(D / P)$ and amplitude are derived. (c) The $D / P$ of conductive network and amplitude of $\Delta R / R_{0}$ peak during dynamic stretching.

connection between CNTs. Therefore, during large deformation process, even if the space between partial CNTs increased, the remaining CNTs could still be in the conductive path through entanglement or FLG connection (blue area), and SCGFs could still exhibit high stability but low response sensitivity. On the whole, SCGFs presented high sensitivity when the CNTs content was equal to their percolation threshold ( $0.30 \mathrm{wt} . \%)$, and high stability when the CNTs content was higher than $0.30 \mathrm{wt} . \%$. The composite fillers played a regulatory role in the strain sensing performance of SCGFs.

To further investigate the dynamic response and stability of SCGFs, the cyclic stretching-releasing tests were carried out at a strain rate of $50 \% \mathrm{~min}^{-1}$ and strain amplitude of $50 \%$. The relation curves between resistance and tensile strain of SCGFs are shown in Fig. 6a. The electrical resistance of SCGFs increases with the increase of tensile strain and decreases with the decrease of tensile strain during the releasing stage. After the first cycle, the relative resistance of SCGFs could not be recovered to its initial value, resulting in a residual relative resistance which decreased with the increase of $m_{\mathrm{CNTS}} / m_{\mathrm{FLG}}$. Some conductive networks were irreversibly destroyed by the irreversible slip of SBS molecular chain (mechanical hysteresis) during stretching. When the CNTs content was higher than their percolation threshold $(0.30 \mathrm{wt} . \%)$, this destructive effect was well restrained. Besides, the $\Delta R / R_{0}$ response of $\mathrm{SBS} / 1 \mathrm{C} / 2 \mathrm{G}$ strain sensors further exhibits excellent stability during 200 stretching-releasing cycles except for the overshoot of incipient cycles due to the viscoelastic nature of SBS matrix (Fig. S3). To quantitatively evaluate the response sensitivity and dynamic stability of SCGFs, the ratio of the drop in relative resistance peaks during tensile cycle tests $(D)$ to the peak value in the first stretch-release cycle $(P)$ was defined as the maximum relative resistance decay $(D / P)$. As shown in Fig. $6 \mathrm{~b}$, a higher $D / P$ indicates a more severe attenuation in strain response sensitivity of composite fibers [38]. According to Fig. $6 c, D / P$ and average amplitude $(A)$ gradually decrease with the increase of $m_{\mathrm{CNT}} / m_{\mathrm{FLG}}$, indicating that the stability of SCGFs conductive network gradually increases and the dynamic response sensitivity decreases. This is consistent with the evolutionary behavior of conductive network as shown in Fig. 5 , confirming again that the higher the $m_{\mathrm{CNTs}} / m_{\mathrm{FLG}}$ is, the more stable conductive network of composite fibers but the lower the strain response sensitivity are. Compared with a single filler, mixed fillers could significantly improve the dynamic stability of the conductive network (lower $D / P$, Table S1), enabling larger deformation.

\section{Effect of applied strain and strain rate on the strain sensing} behavior

The SBS/1C/2G composite fibers were selected to further illustrate the effects of applied strain and strain rate on piezoresistive performances. As shown in Fig. 7a, the relative resistance of SCGFs increases with the increase of tensile strain under different strain amplitudes and the relative resistance gradually decreases during the releas- 

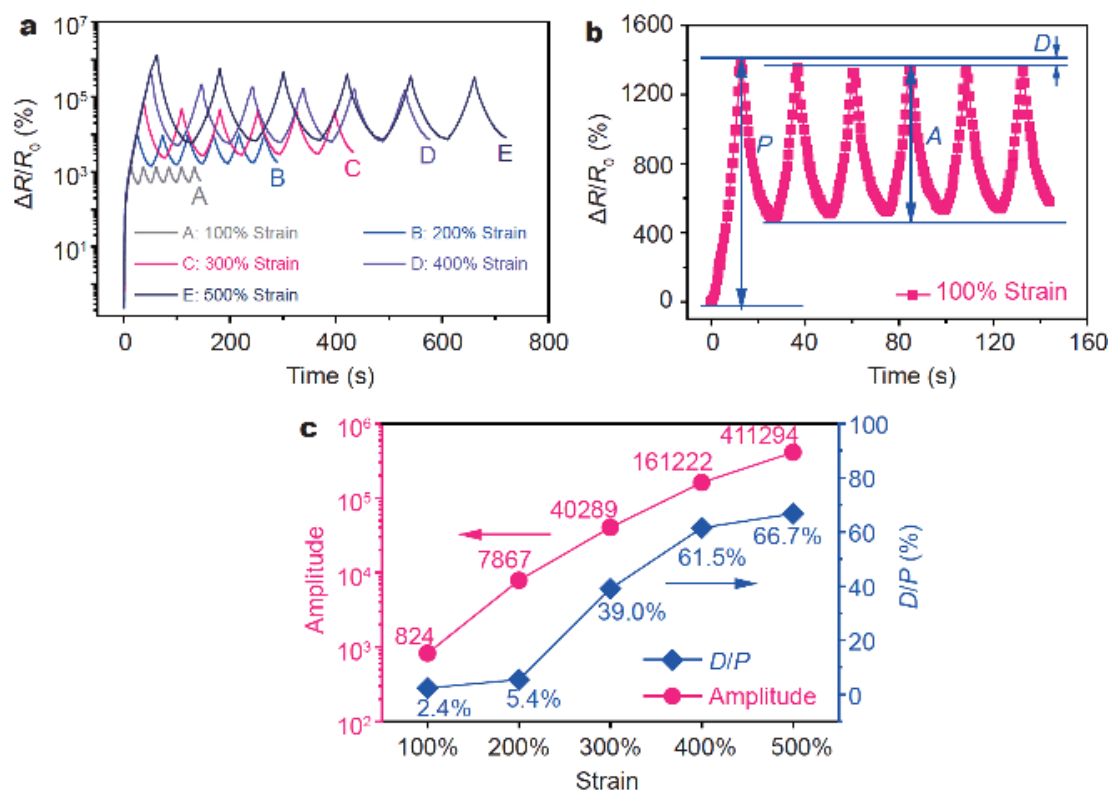

Figure 7 (a) The dynamic strain sensing behavior of SBS/1C/2G fiber with a cyclic loading-unloading of 100\%, 200\%, 300\%, 400\% and 500\%. (b) The representation of where recovery ratio $(D / P)$ and amplitude are derived. (c) The $D / P$ of conductive network and amplitude $\Delta R / R_{0}$ peak during dynamic stretching. Note: The $\Delta R / R_{0}$ is in logarithmic coordinates.

ing process. Moreover, the residual relative resistance of SCGFs varies with the tensile strain amplitude, due to the permanent irreversible structural damage of some conductive networks during stretching process caused by large deformation, such as slippage of SBS molecular chains, slippage between FLG layers, slippage between CNTs and FLG. The relationship between maximum relative resistance decay rate and strain response amplitude of SBS/ $1 \mathrm{C} / 2 \mathrm{G}$ composite fibers at $100 \%$ strain and the relation curves among maximum relative resistance decay rate, strain response amplitude and tensile strain are shown in Fig. 7b, c.

The strain response amplitude increased with the increase of tensile strain amplitude. The deformation (or damage) of the conductive network was more serious when the tensile strain amplitude was greater, leading to an increase in a relative resistance change of SCGFs. In addition, larger strain amplitude led to a more irreversible damage to the conductive network and a higher maximum relative resistance decay rate.

On the contrary, SBS/1C/2G composite fibers exhibited excellent dynamic stability when the tensile strain amplitude was in the range of $0-200 \%$. When the strain was more than $200 \%$, the relative resistance of the composite fibers at the end of the first cycle presented a considerable deduction, and the relative resistance decay rate was $39.0 \%, 61.5 \%$ and $66.7 \%$ with strain amplitude of
$300 \%, 400 \%$ and $500 \%$, respectively. From the second cycle, the strain response of the composite fibers basically reached a stable state. It is important to note that the strain response curves of $S B S / 1 C / 2 G$ composite fibers in the tensile strain range of $100 \%-500 \%$ did not show any unstable "shoulder", suggesting an excellent dynamic stability of the composite fibers. This result is different from the previously reported polymer-based strain sensors, which require multiple cycles to reach a steady state $[22,39]$.

The relationship between the relative resistance and time changes during the stretching-releasing cycles of the SBS/1C/2G composite fibers at a $100 \%$ strain amplitude and a tensile strain rate of $100 \%, 200 \%, 500 \% \mathrm{~min}^{-1}$ is shown in Fig. 8. The strain response sensitivity of the composite fibers is negligibly affected by the tensile strain rate, presenting a good dynamic reliability. With the increase of strain rate, the residual relative resistance of composite fibers increases continuously and the strain response curve "drifts" upward, due to the limited recovery time at a higher stretching-releasing rate and the conductive network could not return to its initial state in a short time.

The piezoresistive mechanism of conductive polymer nanocomposite-based strain sensors is derived from the tunnel effect $[40,41]$. Taking SBS/1C/2G as an example, the tensile strain response of composite fibers was 


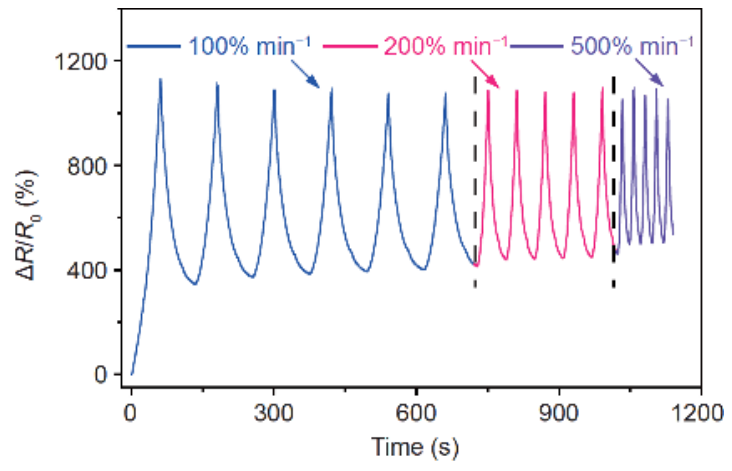

Figure 8 Effect of strain rate on the dynamic sensing performance of $\mathrm{SBS} / 1 \mathrm{C} / 2 \mathrm{G}$ composite fibers.

discussed based on the tunnel effect. The relation between the number of conductive paths $(N)$ and the tensile strain $\varepsilon$ is shown in Equation (2) [42], where $A, B, C$ and $D$ are constants.

$N=\frac{N_{0}}{\exp \left(A \varepsilon+B \varepsilon^{2}+C \varepsilon^{3}+D \varepsilon^{4}\right)}$.

And the relation between the resistance and the tensile strain of the composite fibers could be calculated by Equation (3) [14], where $s$ is the shortest distance between conductive nanoparticles, $b$ is a constant, $\gamma$ is the barrier height between adjacent conductive nanoparticles.

$$
\begin{aligned}
\frac{\Delta R}{R_{0}} & =\frac{R-R_{0}}{R_{0}}=\left[\frac{N_{s}}{N_{0} s_{0}}\right] \exp \left[r\left(s-s_{0}\right)\right]-1 \\
& =(1+b \varepsilon) \exp \left[\left(A+\gamma b s_{0}\right) \varepsilon+B \varepsilon^{2}+C \varepsilon^{3}+D \varepsilon^{4}\right]-1 .
\end{aligned}
$$

Fig. 9 is the fitting of the resistance and tensile strains of SBS/1C/2G based on Equation (2) and (3) where the fitting data and experimental data are highly consistent (correlation index $R^{2}=0.99987$ ). This suggests that theoretical tunnel model can explain the resistance change mechanism of CNTs and FLG hybrid conductive fillers in SCGFs under tensile strains.

\section{CONCLUSIONS}

In this work, the high-performance strain sensors based on 1-D polymer ternary composite fibers (SCGFs) were prepared via wet spinning. The resultant strain sensors simultaneously exhibited high sensitivity, superior stretchability (with a GF of 5,467 under 600\% deformation) and excellent durability, which could be effectively controlled by changing the contents of CNTs and FLG. The SCGF with $0.30 \mathrm{wt} . \%$ CNTs (equal to their percolation threshold $0.30 \mathrm{wt} . \%)$ and $2.7 \mathrm{wt} . \%$ FLG demonstrated the highest sensitivity owing to the bridge

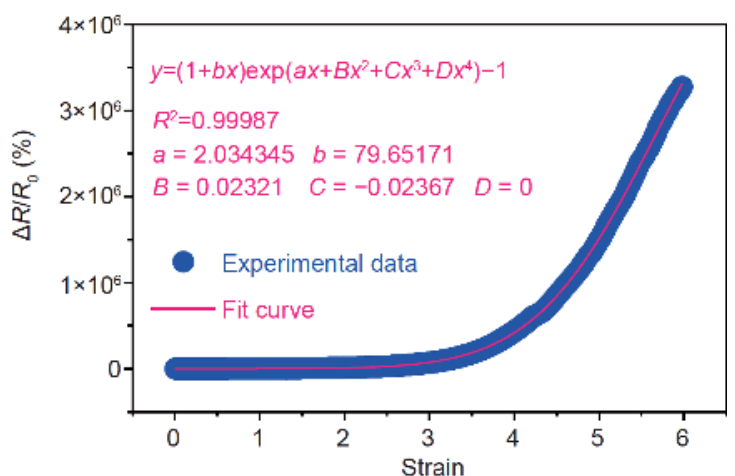

Figure 9 Experimental $\Delta R / R_{0}$ as a function of the applied strain and the fitted curve for the SBS/1C/2G composite fibers.

effect of FLG between adjacent CNTs. The SCGFs with 1.0 wt.\% CNTs (higher than their percolation threshold) and $2.0 \mathrm{wt} . \%$ FLG showed the maximum strain detection range $(600 \%)$ due to the welding connection caused by FLG between contiguous CNTs. In addition, the strain response sensitivity and the maximum relative resistance decay rate of SCGFs also increased with the increase of tensile strain due to the damages of the conductive network during the stretching process. Compared with single-component conductive fillers, such as SBS/CNTs and SBS/FLG composite fibers, the tensile strain detection range of SCGFs was improved. The dynamic stability of SCGFs under large deformation $(100 \%-200 \%)$ was also obviously enhanced. Even if the sensitivity dropped, it still satisfied the requirements for practical applications. Besides, the associated sensing mechanism was also systematically explained by tunneling theory and the evolution of conductive network during stretchingreleasing process. In conclusion, this work provides a simple and efficient assembly method for the preparation of conductive polymer ternary composite fiber-based strain sensors.

\section{Received 17 December 2018; accepted 11 February 2019;} published online 4 March 2019

1 Yamada T, Hayamizu Y, Yamamoto Y, et al. A stretchable carbon nanotube strain sensor for human-motion detection. Nat Nanotechnol, 2011, 6: 296-301

2 Wu H, Liu Q, Du W, et al. Transparent polymeric strain sensors for monitoring vital signs and beyond. ACS Appl Mater Interfaces, 2018, 10: 3895-3901

3 Yu XG, Li YQ, Zhu WB, et al. A wearable strain sensor based on a carbonized nano-sponge/silicone composite for human motion detection. Nanoscale, 2017, 9: 6680-6685

4 Bae SH, Lee Y, Sharma BK, et al. Graphene-based transparent strain sensor. Carbon, 2013, 51: 236-242

5 Kang D, Pikhitsa PV, Choi YW, et al. Ultrasensitive mechanical 
crack-based sensor inspired by the spider sensory system. Nature, 2014, 516: 222-226

6 Park B, Kim J, Kang D, et al. Dramatically enhanced mechanosensitivity and signal-to-noise ratio of nanoscale crack-based sensors: Effect of crack depth. Adv Mater, 2016, 28: 8130-8137

7 Amjadi M, Yoon YJ, Park I. Ultra-stretchable and skin-mountable strain sensors using carbon nanotubes-Ecoflex nanocomposites. Nanotechnology, 2015, 26: 375501-375512

8 Gong S, Lai DTH, Su B, et al. Highly stretchy black gold E-skin nanopatches as highly sensitive wearable biomedical sensors. Adv Electron Mater, 2015, 1: 1400063-1400070

9 Lee J, Kim S, Lee J, et al. A stretchable strain sensor based on a metal nanoparticle thin film for human motion detection. Nanoscale, 2014, 6: 11932-11939

10 Choi DY, Kim MH, Oh YS, et al. Highly stretchable, hysteresis-free ionic liquid-based strain sensor for precise human motion monitoring. ACS Appl Mater Interfaces, 2017, 9: 1770-1780

11 Amjadi M, Pichitpajongkit A, Lee S, et al. Highly stretchable and sensitive strain sensor based on silver nanowire-elastomer nanocomposite. ACS Nano, 2014, 8: 5154-5163

12 Amjadi M, Kyung KU, Park I, et al. Stretchable, skin-mountable, and wearable strain sensors and their potential applications: A review. Adv Funct Mater, 2016, 26: 1678-1698

13 Cheng Y, Wang R, Sun J, et al. A stretchable and highly sensitive graphene-based fiber for sensing tensile strain, bending, and torsion. Adv Mater, 2015, 27: 7365-7371

14 Wang X, Meng S, Tebyetekerwa M, et al. Highly sensitive and stretchable piezoresistive strain sensor based on conductive poly (styrene-butadiene-styrene)/few layer graphene composite fiber. Compos Part A-Appl Sci Manuf, 2018, 105: 291-299

15 Yu S, Wang X, Xiang H, et al. Superior piezoresistive strain sensing behaviors of carbon nanotubes in one-dimensional polymer fiber structure. Carbon, 2018, 140: 1-9

16 Chen S, Wei Y, Yuan X, et al. A highly stretchable strain sensor based on a graphene/silver nanoparticle synergic conductive network and a sandwich structure. J Mater Chem C, 2016, 4: 43044311

17 Yan T, Wang Z, Wang YQ, et al. Carbon/graphene composite nanofiber yarns for highly sensitive strain sensors. Mater Des, 2018, 143: 214-223

18 Lin L, Deng H, Gao X, et al. Modified resistivity-strain behavior through the incorporation of metallic particles in conductive polymer composite fibers containing carbon nanotubes. Polym Int, 2013, 62: 134-140

19 Lin L, Liu S, Zhang Q, et al. Towards tunable sensitivity of electrical property to strain for conductive polymer composites based on thermoplastic elastomer. ACS Appl Mater Interfaces, 2013, 5: 5815-5824

20 Lee S, Shin S, Lee S, et al. Ag nanowire reinforced highly stretchable conductive fibers for wearable electronics. Adv Funct Mater, 2015, 25: 3114-3121

21 Ke K, Pötschke $\mathrm{P}$, Wiegand $\mathrm{N}$, et al. Tuning the network structure in poly(vinylidene fluoride)/carbon nanotube nanocomposites using carbon black: Toward improvements of conductivity and piezoresistive sensitivity. ACS Appl Mater Interfaces, 2016, 8: 14190-14199

22 Liu H, Gao J, Huang W, et al. Electrically conductive strain sensing polyurethane nanocomposites with synergistic carbon nanotubes and graphene bifillers. Nanoscale, 2016, 8: 12977-12989

23 Wang Y, Wang L, Yang T, et al. Wearable and highly sensitive graphene strain sensors for human motion monitoring. Adv Funct Mater, 2014, 24: 4666-4670

24 Zhang X, Feng Y, Tang S, et al. Preparation of a graphene oxidephthalocyanine hybrid through strong $\pi-\pi$ interactions. Carbon, 2010, 48: 211-216

25 Shen B, Zhai W, Chen C, et al. Melt blending in situ enhances the interaction between polystyrene and graphene through $\pi-\pi$ stacking. ACS Appl Mater Interfaces, 2011, 3: 3103-3109

26 Ramanathan T, Abdala AA, Stankovich S, et al. Functionalized graphene sheets for polymer nanocomposites. Nat Nanotechnol, 2008, 3: 327-331

27 Seyedin MZ, Razal JM, Innis PC, et al. Achieving outstanding mechanical performance in reinforced elastomeric composite fibers using large sheets of graphene oxide. Adv Funct Mater, 2015, 25: 94-104

28 Costa P, Ferreira A, Sencadas V, et al. Electro-mechanical properties of triblock copolymer styrene-butadiene-styrene/carbon nanotube composites for large deformation sensor applications. Sensor Actuat A-Phys, 2013, 201: 458-467

29 Ma LF, Bao RY, Dou R, et al. Conductive thermoplastic vulcanizates (TPVs) based on polypropylene (PP)/ethylene-propylenediene rubber (EPDM) blend: From strain sensor to highly stretchable conductor. Compos Sci Tech, 2016, 128: 176-184

30 Park SJ, Kim J, Chu M, et al. Highly flexible wrinkled carbon nanotube thin film strain sensor to monitor human movement. Adv Mater Technol, 2016, 1: 1600053-1600061

31 Kim I, Woo K, Zhong Z, et al. A photonic sintering derived Ag flake/nanoparticle-based highly sensitive stretchable strain sensor for human motion monitoring. Nanoscale, 2018, 10: 7890-7897

32 Coskun MB, Akbari A, Lai DTH, et al. Ultrasensitive strain sensor produced by direct patterning of liquid crystals of graphene oxide on a flexible substrate. ACS Appl Mater Interfaces, 2016, 8: 2250122505

33 Wang C, Xia K, Jian M, et al. Carbonized silk georgette as an ultrasensitive wearable strain sensor for full-range human activity monitoring. J Mater Chem C, 2017, 5: 7604-7611

34 Li YQ, Huang P, Zhu WB, et al. Flexible wire-shaped strain sensor from cotton thread for human health and motion detection. Sci Rep, 2017, 7: 45013-45020

35 Costa P, Silva J, Ansón-Casaos A, et al. Effect of carbon nanotube type and functionalization on the electrical, thermal, mechanical and electromechanical properties of carbon nanotube/styrenebutadiene-styrene composites for large strain sensor applications. Compos Part B-Eng, 2014, 61: 136-146

36 Boland CS, Khan U, Backes C, et al. Sensitive, high-strain, highrate bodily motion sensors based on graphene-rubber composites. ACS Nano, 2014, 8: 8819-8830

37 Ryu S, Lee P, Chou JB, et al. Extremely elastic wearable carbon nanotube fiber strain sensor for monitoring of human motion. ACS Nano, 2015, 9: 5929-5936

38 Duan L, Fu S, Deng H, et al. The resistivity-strain behavior of conductive polymer composites: Stability and sensitivity. J Mater Chem A, 2014, 2: 17085-17098

39 Lin Y, Liu S, Chen S, et al. A highly stretchable and sensitive strain sensor based on graphene-elastomer composites with a novel double-interconnected network. J Mater Chem C, 2016, 4: 63456352

40 Lee C, Jug L, Meng E. High strain biocompatible polydimethylsiloxane-based conductive graphene and multiwalled carbon nanotube nanocomposite strain sensors. Appl Phys Lett, 
2013, 102: 183511-183516

41 Zhao J, He C, Yang R, et al. Ultra-sensitive strain sensors based on piezoresistive nanographene films. Appl Phys Lett, 2012, 101: 063112

42 Sheng P, Sichel EK, Gittleman JI. Fluctuation-induced tunneling conduction in carbon-polyvinylchloride composites. Phys Rev Lett, 1978, 40: 1197-1200

Acknowledgements This work was supported by the Fundamental Research Funds for the Central Universities (2232018D3-03 and 2232018A3-01), the Program for Changjiang Scholars and Innovative Research Team in University (IRT16R13), the National Natural Science Foundation of China (51603033), the Science and Technology Com- mission of Shanghai Municipality (16JC1400700) and the Innovation Program of Shanghai Municipal Education Commission (2017-01-0700-03-E00055).

Author contributions $\mathrm{Zhu} \mathrm{M}$ and Xiang $\mathrm{H}$ supervised the project. $\mathrm{Yu}$ $S$ designed and performed the experiments, analyzed the data and wrote the manuscript with support from Wang X and Tebyetekerwa M. All authors contributed to the general discussion.

Conflict of interest The authors declare no conflict of interest.

Supplementary information Supporting data are available in the online version of the paper.

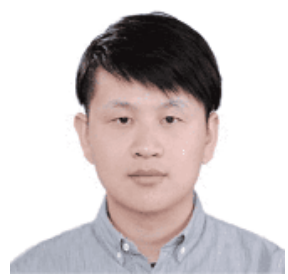

Senlong Yu is now a PhD candidate in Prof. Meifang Zhu's group at the College of Materials Science and Engineering, Donghua University. His current research focuses on the smart fiber materials.
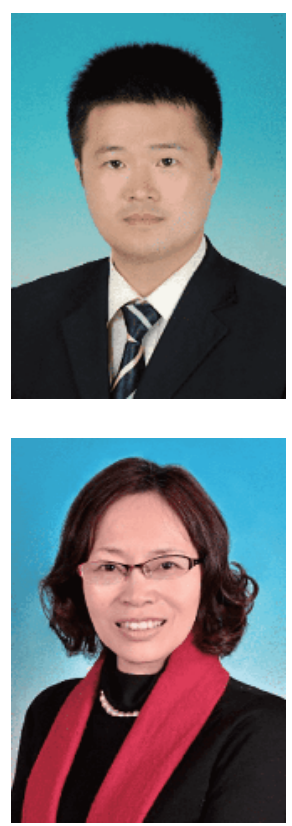

Hengxue Xiang is an assistant research fellow of Donghua University. He obtained his $\mathrm{PhD}$ at the College of Materials Science and Engineering, Donghua University. His research focuses on the fiber formation of bio-based fibers and intelligent fibers.

\section{基于三元复合导电纤维应变传感器的构筑及其性能研究}

俞森龙, 王兴平, 相恒学*, Mike Tebyetekerwa, 朱美芳*

摘要 针对现有导电高分子基应变传感材料无法兼顾传感性、拉伸性和稳定性的难题, 我们采用石墨烯(FLG)和碳纳米管(CNT)为复合导 电填料、以聚(苯乙烯-丁二烯-苯乙烯)(SBS)为柔性基体，利用湿法纺丝成形技术制备了基于SBS/CNT/FLG的三元复合导电纤维 (SCGFs) 应变传感器. 系统研究了不同导电材料的含量与比例、应变幅度与速率等条件下材料传感性能的变化. 结果显示: SCGFs基应变传感器兼 具高灵敏性 (GF $>5000)$ 、宽应变范围( $>600 \%)$ 与循环稳定性 $(>200$ cycles), 同时可通过改变CNT和FLG添加量实现材料传感性能的精确调 控, 这主要归因于SBS良好的拉伸-回复性能、材料之间较强的 $\pi-\pi$ 相互作用以及复合填料的协同效应. 此外, 我们通过分析拉伸-释放循环 实验中SCGFs复合导电纤维导电网络的演变验证了其应变传感机理符合隧道效应理论模型. 